\title{
Personas con Discapacidad y Aprendizaje Virtual: Retos para las TIC en Tiempos de Covid-19
}

\author{
People with Disabilities and Virtual Learning: Challenges for ICT in the Days of Covid- \\ 19
}

\author{
Claudia Cintya Peña-Estrada ${ }^{1}$,Milady Vaillant-Delis ${ }^{2}$, Osmanys Soler-Nariñoo ${ }^{3}$,Yaser Bring-Pérez $z^{4}$ y Yinet \\ Domínguez-Ruiz ${ }^{5}$
}

\section{0 \\ EDICIÓN: 粃-CIVTAC}

Recibido: 19/julio/2020

Aceptado: 30/agosto/2020

Publicado: 25/septiembre/2020

${ }^{1}$ México
${ }^{2} \mathrm{Cuba}$
${ }^{3} \mathrm{Cuba}$
${ }^{4} \mathrm{Cuba}$
${ }^{5} \mathrm{Cuba}$

\section{IIIInstitución}

${ }^{1}$ Universidad Autónoma de Querétaro ${ }^{2}$ Universidad de Oriente

${ }^{3}$ Universidad de Oriente

${ }^{4}$ Universidad de Oriente

${ }^{5}$ Universidad de Oriente

\section{Correo Eletrónico}

1'claudia.cintya.pena@uaq.mx

2milo@uo.edu.cu

3osoler@uo.edu.cu

${ }^{4}$ yaser@uo.edu.cu

5 yinetd94@gmail.com

\section{iDORCID}

${ }^{1}$ https://orcid.org/0000-0003-0378-0762 ${ }^{2}$ https://orcid.org/0000-0002-0688-6960 ${ }^{3} \mathrm{https} / / /$ orcid.org/0000-0001-8239-5306 ${ }^{4}$ https://orcid.org/0000-0001-5184-9678 ${ }^{5}$ https://orcid.org/0000-0001-6403-6465

\section{Citar así: CGAPA / IEEE}

Peña-Estrada, C., Vaillant-Delis, M., Soler-Nariño, O., Bring-Pérez, Y. \& Domínguez-Ruiz, Y. (2020). Personas con Discapacidad y Aprendizaje Virtual: Retos para las TIC en Tiempos de Covid-19. Revista Tecnológica-Educativa Docentes 2.0, 9(2), 204-211. https://doi.org/10.37843/rted.v9i2.165

C. Peña-Estrada, M. Vaillant-Delis, O. Soler-Nariño, Y. Bring-Pérez y Y. Domínguez-Ruiz, "Personas con Discapacidad y Aprendizaje Virtual: Retos para las TIC en Tiempos de Covid-19", RTED, vol. 9, n. ${ }^{\circ}$, pp. 204-211, sep. 2020 .

\section{Resumen}

El presente artículo resalta la importancia de crear entornos educativos más accesibles a las personas con discapacidad (PCD) dentro de los Objetivos de Desarrollo Sostenible (ODS). Cumplir con estos ODS, en especial el cuatro (educación inclusiva) constituye un desafío en situaciones de aislamiento. Asimismo, la Convención de los Derechos de las Personas con Discapacidad (2006), reconoce el derecho a la información y comunicación (incluidas las Tecnologías de la Información y la Comunicación (TIC)). Sin embargo, alcanzar el aprendizaje virtual en ese colectivo, emerge como un reto significativo en tiempos de Covid-19. Con el cierre de las escuelas derivado de esta pandemia, se generan nuevos espacios de aprendizaje que requieren habilidades, conocimientos y medios tecnológicos. En este sentido, el objetivo propuesto se dirige a valorar los principales desafíos que presenta el aprendizaje virtual en las PCD a través de las TIC en tiempos de pandemia. La metodología empleada estuvo sustentada en métodos generales del conocimiento científico en función de analizar y sintetizar diversas concepciones teóricas sobre discapacidad. También se aplicó el análisis de contenido para profundizar en el conocimiento legitimado en torno a esta temática. Los resultados obtenidos destacan las estrategias desarrolladas en Cuba para el aprendizaje inclusivo (programas educativos hacia todos los discapacitados). No obstante, persisten insuficiencias en el ajuste de estos espacios debido a brechas tecnológicas y representaciones que signan el aprendizaje en tiempos de Covid-19. Se necesita introducir una concepción sociocultural compleja del aprendizaje virtual donde se adapten los recursos tecnológicos a esas características socioculturales individuales o colectivas.

Palabras clave: Aprendizaje virtual, personas con discapacidad, inclusivo educativa, TIC, Covid-19.

\begin{abstract}
This article highlights the importance of creating educational environments more accessible to people with disabilities (PWD) within the Sustainable Development Goals (ODS). Meeting these SDGs, especially four (inclusive education) is a challenge in situations of isolation. Likewise, the Convention on the Rights of Persons with Disabilities (2006) recognizes the right to information and communication (including Information and Communication Technologies (ICT)). However, achieving virtual learning in this group emerges as a significant challenge in times of Covid-19. With the closure of schools derived from this pandemic, new learning spaces are generated that require skills, knowledge, and technological means. In this sense, the proposed objective is aimed at assessing the main challenges that virtual learning presents in PWD through ICT in times of pandemic. The methodology used was supported by general methods of scientific knowledge to analyze and synthesize various theoretical conceptions about disability. Content analysis was also applied to deepen the legitimized knowledge around this topic. The results obtained highlight the strategies developed in Cuba for inclusive learning (educational programs for all disabled people). However, inadequacies persist in the adjustment of these spaces due to technological gaps and representations that signify learning in times of Covid-19. It is necessary to introduce a complex sociocultural conception of virtual learning where technological resources are adapted to those individual or collective sociocultural characteristics.
\end{abstract}

Keywords: Virtual learning, people with disabilities, inclusive education, ICT, Covid-19. 
Según la Organización Mundial de la Salud (OMS) y la Organización de Naciones Unidas (ONU) las cifras de personas con discapacidad a nivel internacional se ubican, aproximadamente, en el 15\% de la población mundial (OMS, 2011 \& ONU, 2011). En el caso de América Latina y el Caribe, organismos regionales como la Comisión Económica para América Latina y el Caribe (CEPAL) afirman que más de 70 millones de estas personas se exponen de manera diaria a situaciones de discriminación matizadas por brechas socioeconómicas, de género, edad, lugar de residencia, su condición étnico-racial y el estatus migratorio, entre otras (CEPAL, 2014). Con la irrupción de la Covid-19 en el contexto global, dichas brechas se acentúan o ensanchan para este grupo social, pues la mayoría de ellas se encontraban en condiciones de exclusión, marginalidad y estigmatización antes de la pandemia.

La debilidad de los sistemas de salud a escala global y regional para enfrentar los efectos de la pandemia han conllevado a contradicciones políticas relacionadas con la desprotección a los grupos sociales más vulnerables, así como a individuos y familias con mayores dificultades, por ejemplo, en el acceso al empleo, seguridad social, educación, alimentación, vivienda y educación (Huenchuan, 2020). En este último los impactos de la Covid-19 dejarán importantes secuelas en el desarrollo de una educación inclusiva en muchos países. Datos ofrecidos por la CEPAL (2020) señalan que, en el mes de marzo, alrededor de ciento trece (113) millones de niños, niñas y adolescentes se encontraban, fuera de las escuelas, cerradas a causa de la COVID-19. Esto evidencia los desafíos que experimentan muchos sistemas educativos para lograr un aprendizaje inclusivo en el contexto de la pandemia, pues los escenarios docentes se trasladaron a las viviendas en condiciones no siempre favorables para la enseñanza.

En América Latina y el Caribe muchos hogares se enfrentan a riesgos sanitarios, alimentarios, de género, económicos, de empleo, culturales, entre otros, que se interconectan con la fragilidad de las relaciones sociales, los escasos recursos y activos educativos en función de desarrollar una educación inclusiva y de calidad. Esta compleja situación de vulnerabilidad se complejiza en las personas con discapacidad, quienes deben, por un lado, superar las estructuras simbólicas o mentales que legitiman los estigmas sociales en torno a su condición de discapacidad, y por el otro lado, acceder a entornos educativos con barreras físicas y bajo condiciones no adecuadas a la diversidad funcional de estas personas.

Al respecto, la Agenda 2030 para el desarrollo sostenible en su objetivo 4 y meta 4.7(a) plantea la necesidad de construir y adecuar los espacios educativos a las necesidades de los niños y las personas con discapacidad, lo que incluye infraestructuras y materiales adaptados a esos estudiantes (CEPAL, 2018, p.29). De igual manera, la Convención sobre los Derechos de las Personas con Discapacidad (CDPCD) refrenda la necesidad de eliminar la vulnerabilidad y desigualdad social hacia este grupo socia, a través de potenciar la igualdad de oportunidades y acceso sin barreras a espacios educativos más inclusivos y participativos.

Por consiguiente, en condiciones de aislamiento derivadas de la Covid-19, este colectivo requiere mayores adaptaciones y ajustes para lograr su inclusión educativa ante las transformaciones sociales y económicas que ha generado la pandemia. Todo ello constituye un reto en los marcos de la Agenda 2030 y la CDPCD, pues todavía existen fragilidades físicas, económicas, sociales y culturales que impiden la participación de las personas con discapacidad, en los nuevos escenarios educativos dentro de la actual crisis, por ejemplo, el aprendizaje virtual.

En este sentido, autores como Camacho \& Varela (2011); Lancheros, Carillo \& Lara (2011); Silvana \& Andrea (2016); Mogollón, Medina \& Correa (2017) abordan que existen brechas digitales en el acceso a las computadoras, Internet y las habilidades en el empleo de estos dispositivos y plataformas virtuales por las personas con discapacidad. Según estos investigadores se necesita de nuevas adaptaciones a ese aprendizaje virtual, pero desde concepciones más inclusivas e integradoras. Asimismo, otras investigaciones sostienen la reducción de esa brecha digital a partir del diseño o ajuste de las Tecnologías de la Información y la Comunicación (TIC), con el fin de alcanzar mayor inclusión social (Flórez et al., 2016).

En las personas con discapacidad esta inclusión se asocia, entre otros elementos, a la autonomía personal, la reducción de las desigualdades y las barreras del entorno que generan situaciones de dependencia ante los demás. Dicho entorno social (o digital) produce estructuras que lejos de incluir, excluyen de la participación y la realización de esa autonomía personal. 
La perspectiva anterior es defendida por Pérez (2010); Barnes (2010); Jiménez \& Huete (2010); Bernabeu (2012); Agulló \& Arcas (2012); Alcaín \& Álvarez (2015); Díaz (2018), quienes se enfocan en la reducción y eliminación de las estructuras físicas, sociales y económicas que impiden el desarrollo humano de las personas con discapacidad.

En Cuba, la concepción de inclusión comprende el respeto a los derechos en función de que estas personas puedan ejercerlos y disfrutarlos plenamente. La política social cubana asume ese enfoque inclusivo con el respaldo de su Constitución y las normas, leyes, reglamentos y resoluciones específicas creadas por el Estado (García \& Tamayo, 2018). Cada uno de estos instrumentos jurídicos orientan sus esfuerzos a una mayor accesibilidad del entorno y no discriminación para este grupo poblacional, a partir de reducir o eliminar esas barreras sociales, culturales o físicas de ese contexto estructural.

Con la actualización del modelo económico y social cubano, el Plan Nacional de Desarrollo Económico y Social hasta el 2030 introduce objetivos dirigidos a eliminar la vulnerabilidad y desigualdad social de las personas con discapacidad. Por ejemplo, en el eje estratégico relacionado con el desarrollo humano, equidad y justicia social se evidencian dos objetivos (16 y 19) que signan estas ideas. En el primero se señala la importancia del diseño de políticas diferenciadas para la atención de los grupos con necesidades especiales. El segundo apunta la necesidad de asegurar la igualdad de oportunidades y la garantía de acceso para impulsar la inclusión económica, política y social, así como proteger aquellas en condiciones de vulnerabilidad.

Desde el contexto cubano, la educación hacia las personas con discapacidad potencia el desarrollo de sus habilidades, capacidades y conocimientos en interacción con el medio social (García \& Tamayo, 2018). Este entorno debe contribuir a la socialización e igualdad de oportunidades con la finalidad de lograr mayor inclusión y participación de ese grupo social en los espacios virtuales de aprendizaje.

Sin embargo, el diseño de un entorno de aprendizaje y conocimiento relacionado con las Tecnologías de la Información y la Comunicación (TIC) en el contexto actual, se encuentra matizado por complejas situaciones sociales y económicas originadas por la pandemia. Con el cierre de escuelas, la responsabilidad educativa, acompañamiento y apoyo de los padres adquiere relevancia para alcanzar ese aprendizaje virtual dentro de ambientes familiares desiguales en cuanto a las habilidades, activos o recursos que permitan atender las características socioeducativas diversas de sus hijos e hijas con discapacidad.

En este sentido, el objetivo de la presente ponencia se dirige a valorar los principales desafíos que presenta el aprendizaje virtual mediante las TIC para las PCD en tiempos de pandemia, municipio Santiago de Cuba, Cuba. Para materializar este objetivo se introdujo una perspectiva metodológica sustentada en métodos generales del conocimiento científico y el análisis de contenido, en función de analizar los desafíos vinculados con las brechas tecnológicas en condiciones de confinamiento social. Consideramos importante adoptar no solo nuevos ambientes de aprendizaje en los que se utilicen las TIC en igualdad de condiciones para todos, sino también una concepción más inclusiva de la discapacidad hacia la eliminación de las brechas y desafíos digitales acentuados por la pandemia Covid-19.

Esta situación ubica nuestro aporte investigativo en el plano teórico y metodológico, pues se introduce una concepción relacional de ese grupo social en su aprendizaje virtual. Desarrollar este enfoque requiere concebir a la discapacidad como una construcción sociocultural compleja, resultado de las interrelaciones o interconexiones entre la cultura normativa (normas y valores), organización social (tecnología digital) y la particularidad diversa (habilidades y conocimiento) de esas personas. Junto a esas interacciones también se configuran los diferentes procesos de atención a problemáticas que afectan a la discapacidad, entre ellas, la inclusión educativa mediante la adecuación de las TIC a las características de este colectivo social.

\section{Metodología}

En el desarrollo de la investigación se utilizaron métodos del nivel teórico (análisis, síntesis, inducción y deducción), así como el análisis de contenido con el objetivo de profundizar en el conocimiento legitimado en torno a la discapacidad en el contexto internacional y nacional. La descomposición analítica de las diferentes concepciones teóricas en torno a la discapacidad permitió sintetizarlas y delimitar sus principales limitaciones en la comprensión de un enfoque más 
integrado para alcanzar el aprendizaje virtual a través de las TIC. Esto condujo a la concepción de la discapacidad como construcción sociocultural compleja, enfoque sintetizador de los factores culturales y sociales en la inclusión educativa de este grupo poblacional.

Mediante la inducción y deducción se pudo obtener una caracterización de la población con discapacidad en Cuba, así como su comportamiento en el territorio. La recogida de información a través del análisis de contenido a la bibliografía relacionada con el tema muestra la importancia de lograr entornos educativos más inclusivos y adaptados a realidad simbólica de estas personas. Desde esa perspectiva, la construcción sociocultural compleja expresa la adecuación de los espacios virtuales de aprendizaje a dichas personas, atendiendo a sus características físicas, visuales, diversidad intelectual y sensorial.

\section{Resultados}

La concepción de la discapacidad como construcción sociocultural compleja, conlleva a posiciones teóricas que resaltan las estructuras sociales, culturales y económicas en la configuración de la situación de discapacidad. Ferrante \& Ferreira (2010) afirman que la discapacidad adquiere sentido en el contexto de un sistema cultural determinado, emergiendo como construcción interpretativa legitimada por éste. Con referencia a lo anterior, se valora la introducción de esa dimensión sociocultural como estructura sociosimbólica que configura los procesos de inclusión social de estas personas.

Discursos más cercanos a los derechos sociales y políticos de este colectivo (Venturiello, 2017; Díaz, 2018; Pérez \& Chabra, 2019), sustentan la construcción sociohistórica de la discapacidad mediante la ruptura de las barreras que limitan el acceso al entorno, igualdad de oportunidades, el respeto a las diferencias y la participación social en el diseño de políticas públicas más inclusivas hacia esas personas.

Desde la diversidad, los investigadores Palacios \& Romañach (2006) proponen una nueva perspectiva a partir del funcionamiento diverso de la naturaleza humana a nivel social. Según esta perspectiva las personas con discapacidad conforman un grupo social con comportamientos, interacciones, comunicaciones, sistemas simbólicos, procesos de socialización, biografía individual y colectiva, constitutivos de su diversidad e inclusión educativa.

Posturas teóricas recientes abordan a la discapacidad como construcción cultural conformada por estructuras de discriminación, marginación y exclusión que configuran la situación de estas personas (González, 2010). Dichas posiciones teóricas destacan los constructos simbólicos que subyacen en la construcción interpretativa de la discapacidad y su configuración relacional a partir de la estructura social constituida en cada sociedad. También se incluyen las barreras que excluyen, discriminan o estigmatizan a este grupo social en el acceso a programas educativos, las TIC y el aprendizaje virtual.

Otro estudio interesante en torno a la discapacidad es el enfoque sociológico de Brogna (2012) sobre la encrucijada social. En la construcción teórica de esta encrucijada intervienen tres elementos que interactúan entre sí, generando situaciones de vulnerabilidad social. Ellos son: la cultura normativa, organización socioeconómica y particularidad biológica-conductual del actor individual o colectivo. Aplicadas al objeto de estudio la cultura normativa hace referencia al sistema cultural que signa o legitima el acceso de estas personas al aprendizaje virtual. Dicha cultura normativa está compuesta por representaciones, significaciones, ideas, creencias, saberes, normas o valores, entre otros. Estos elementos conforman el capital cultural que estigmatiza las posibilidades de participar y hacer uso de las TIC. Por ejemplo, la cultura normativa sitúa a esos individuos en sujetos de ayuda, sobreprotección o vulnerables para acceder a esas tecnologías.

Desde la perspectiva compleja la cultura normativa se interconecta con la organización económica y social (donde se ubican las TIC). Aquí están los medios tecnológicos que permiten la inclusión educativa a través de nuevos ambientes digitales. Esta organización está integrada por instituciones, plataformas virtuales, familias y grupos que participan de este proceso de integración a través de las TIC.

Por último, la particularidad biológicoconductual comprende las características individuales o colectivas de estas personas, sus prácticas, imaginarios culturales y los significados construidos en su modo de vida. Todo lo anterior conforma la heterogeneidad social necesaria para adaptar los ambientes virtuales o las TIC a dicha particularidad, y no al revés, las personas con discapacidad deben 
ajustarse a la cultura normativa de los espacios virtuales de aprendizaje.

La interconexión de estos tres elementos genera la construcción sociocultural compleja para analizar el tema de la inclusión educativa mediante las TIC. Algunos autores apuntan que alcanzar la inclusión social implica, primero, el acceso a la ciudadanía y a los derechos económicos, políticos y sociales. Segundo, poseer sólidas redes de reciprocidad social (ejemplo familiar, afectivo, comunitario, entre otras). Tercero, mayor integración al espacio de producción económica, fundamentalmente el mercado de trabajo (Subirats et al., 2009, p.4). En el caso de las personas con discapacidad, la inclusión educativa a través de las TIC implica tener redes sociales que faciliten el acceso a recursos tecnológicos para desarrollar el aprendizaje virtual. En Cuba las asociaciones de personas con discapacidad, la familia y los docentes conforman un entramado importante de relaciones para desarrollar el aprendizaje virtual ante carencias en materia de activos digitales en situaciones de confinamiento social.

Una inclusión transformadora debe insertar instituciones $y$ organizaciones en función de propiciar la participación plena de este grupo poblacional en las actividades de su comunidad (Samaniego, 2006, p.33). Esto favorece el acceso a las TIC e inclusión educativa como un acceso sin barreras al aprendizaje, comunicación o información en el entorno digital (Gómez et al., 2006). Esta afirmación reconoce el derecho a la no discriminación, así como la adopción de medidas para garantizar igualdad de oportunidades en esos ambientes digitales.

Para Habermas (2012) la inclusión se materializa en esa sensibilidad social hacia las particularidades individuales o colectivas de los excluidos. Dicha concepción se encamina al reconocimiento de las características socioculturales del grupo poblacional con discapacidad. Además, la protección de sus derechos en igualdad de condiciones al resto de miembros de la sociedad y el respeto a su diversidad.

Este último enfoque, junto con el de Brogna (2012), aportan una estructura teórica que permite concebir a la discapacidad como construcción sociocultural compleja, pues ésta es resultado de la interacción e interrelación entre cultura normativa, organización socioeconómica y la particularidad social diversa como elemento constitutivo de estas personas con discapacidad. La relación que establecen estas tres categorías es transversalizada por la inclusión social cuyo contenido epistémico está en crear una sensibilidad capaz de eliminar barreras, discriminaciones, estigmas o vulnerabilidades.

La discapacidad como construcción sociocultural compleja permite la aproximación a los conflictos y contradicciones producidas, entre los elementos citados, en esos ambientes digitales. Para lograr la inclusión educativa en el tema discapacidad, la cultura normativa con su carga simbólica, así como los actores sociales que producen y reproducen dicha cultura se adaptan a esas particularidades físicas, sensoriales, intelectuales, de habilidades o aprendizaje de las personas con discapacidad. Esta adaptación incluye a la organización socioeconómica como generadora de tecnologías encaminadas a la participación e incorporación de todos a una comunidad de aprendizaje virtual diverso.

\section{Las Personas con Discapacidad en el Aprendizaje Virtual: Desafíos en Tiempos de Pandemia}

En su decurso histórico el desarrollo tecnológico introdujo nuevos escenarios de aprendizaje (por ejemplo, el virtual) que transformaron los ámbitos tradicionales de enseñanza. Junto a este desarrollo acelerado de los recursos tecnológicos, emergieron desigualdades y vulnerabilidades en el acceso al conocimiento de manera virtual. Aquí destaca la adaptación de estos entornos de aprendizaje virtual a la particularidad social diversa de las personas con discapacidad en condiciones de aislamiento social.

La irrupción de la pandemia Covid-19, como hecho social total, transformó la dinámica social tradicional de principios del 2020, por tanto, se necesitan nuevas formas de resiliencia social para adaptarse a una etapa post pandemia. En el campo educativo, la cultura normativa en materia digital requiere incluir las necesidades de esas personas con discapacidad en función de superar los obstáculos en el orden de la comunicación, comprensión o movilidad (Mogollón, Medina \& Correa, 2017).

En los marcos de los ODS y la CDPCD, se generan desafíos relacionados con el acceso a las tecnologías de la información y comunicación para 
este colectivo social en en condiciones de aislamiento. Informes recientes de la CEPAL y la Corporación Andina de Fomento (2020, p.10), advierten que existen brechas tecnológicas en diversos grupos o segmentos poblacionales hacia un desarrollo adecuado del aprendizaje virtual. De ahí la importancia de seguir impulsando políticas públicas de conectividad e inversión en infraestructura digital.

Ajustar la organización socioeconómica (tecnológica) a esa realidad social de las personas con discapacidad en ambientes desiguales y vulnerables, pone en riesgo el cumplimiento de los ODS en relación con una educación de calidad, inclusiva e igualitaria a todos los niveles (preescolar, primaria, secundaria, universitaria, entre otras). Igualmente, los impactos de la Covid-19 ensancharán las disparidades para estas personas en el acceso al aprendizaje virtual mediante el empleo de los medios tecnológicos.

En el caso de la CDPCD, sus retos también son medibles, pues alcanzar mayor accesibilidad de los entornos virtuales será una tarea compleja para la organización socioeconómica actual. Aunque se han logrado avances en los servicios de información y comunicación, todavía es insuficiente el ajuste de la cultura normativa que orienta a los nuevos sistemas tecnológicos de información y comunicación. Es necesario priorizar los ajustes razonables como modificaciones o adaptaciones a esos entornos digitales (incluidas las TIC), en función de la particularidad social diversa de estos individuos hacia una educación de calidad e inclusiva (Acosta et al., 2020).

En Cuba, la educación está concebida como un derecho de todos y sus resultados evidencian logros vinculados no solo con el Informe Mundial Enseñanza y Aprendizaje, sino también al Objetivo 4 de la Agenda 2030. En este sentido la educación cubana, en los últimos años, alcanzó un significativo índice de desarrollo educacional (IDE 0.983) a pesar del reforzamiento del bloqueo económico y financiero de Estados Unidos.

En Cuba, la inclusión educativa exige el ajuste del modelo de escuela actuante, lo que implica profesores distintos, padres diferentes, así como una organización escolar integrada a la comunidad. La cultura normativa inserta en las escuelas especiales del territorio ha sido capaz de priorizar la particularidad social diversa de ese grupo, construyendo entornos educativos más saludables en cuanto, a su accesibilidad, formación de habilidades o capacidades.

No obstante, si bien el aprendizaje en tiempos de pandemia está diseño para su realización a través medios audiovisuales, no todas las familias pueden obtener de manera digitalizada estos productos televisivos. En las personas con discapacidad esta situación se interconecta con esa construcción social compleja conformada por relaciones entre la organización socioeconómica, cultura normativa y la particularidad social diversa. Por ejemplo, aunque muchas clases tienen servicios de traducción para esa particularidad diversa (discapacidad auditiva), la interacción social se disminuye en situaciones de aislamiento, elemento fundamental para alcanzar mayor participación de estas personas en la sociedad.

En este sentido se reduce el acceso a los recursos o activos tecnológicos que permiten, después de finalizada estas clases virtuales, la reproducción del contenido aprendido a través de la interacción social o socialización. La cultura normativa expresada en valores y normas de comunicación digital a veces no permite que muchos de estos grupos adquieran ese conocimiento, pues en ocasiones su diseño está escasamente adaptado a su particularidad diversa. A estas situaciones de vulnerabilidad, se incorporan en muchos ambientes familiares de personas con discapacidad, el bajo capital económico para acceder a redes de comercialización tecnológica en condiciones de pandemia.

\section{Conclusiones}

Las investigaciones abordadas posibilitaron analizar el aprendizaje virtual para las personas con discapacidad en tiempos de pandemia a partir de la interacción e interconexión entre cultura normativa, organización socioeconómica y esa particularidad social diversa(biológica-conductual) característica del grupo social estudiado. Estos elementos configuran en sus interrelaciones, la construcción sociocultural compleja en el tema discapacidad.

En esta concepción compleja se muestra la integración macro-micro en el análisis a la inclusión educativa mediante el empleo de las TIC. Dicha situación evidencia el entramado de relaciones establecidas entre la dimensión simbólica inscrita en la discapacidad y esas dinámicas estructurales del sistema social. Lo anterior permite encontrar los conflictos o contradicciones que emergen durante la 
adecuación de estas tecnologías a los ambientes digitales de aprendizaje.

El uso de las TIC en tiempos de Covid-19 requiere fortalecer las redes, vínculos sociales, estructura de oportunidades, así como los activos y recursos en función de alcanzar mayor accesibilidad a través de estas tecnologías. Para ello, todos los actores sociales (familiares, docentes, estudiantes, entre otros) deben utilizar sus recursos tangibles e intangibles como: los valores, capital cultural, saberes, habilidades y conocimientos hacia la inclusión de esas personas con discapacidad en los espacios de aprendizaje virtual.

Los resultados obtenidos evidencian que estos individuos enfrentan barreras mentales y físicas en el acceso a los nuevos entornos de enseñanza en tiempo de pandemia. Esta condición acentúa las brechas tecnológicas existentes, todavía, a nivel familiar para lograr un aprendizaje virtual más sensible a las diferencias individuales o colectivas del trasfondo cultural. Al respecto, se considera necesario generar acciones públicas que, primero, caractericen a estas personas en cuanto a sus habilidades o capacidades para el aprendizaje virtual, según el tipo de discapacidad (físico-motora, ciego, sordo, intelectual, entre otras). Segundo, diagnosticar quiénes poseen recursos tecnológicos y las condiciones físicas de éstos. Tercero, focalizar a los que no tienen esos recursos en función de lograr un aprendizaje virtual más inclusivo y participativo.

\section{Referencias}

Acosta, M., Betún, A., Delgado, J., \& Iñiguez, M. (2020). Las TIC como oportunidad para fortalecer el PEA en los estudiantes con discapacidad visual. Revista Tecnológica-Educativa Docentes 2.0, 9(1), 42-48. https://doi.org/10.37843/rted.v9i1.9

Agulló, C. \& Arcas, I. (2012). Una mirada hacia los colectivos vulnerables: personas mayores y personas con discapacidad en la acción humanitaria. Edita Instituto de Estudios sobre Conflictos y Acción Humanitaria (IECAH).

Alcaín, E. \& Álvarez, G. (2015). La convención internacional sobre los derechos de las personas con discapacidad. De los derechos a los hechos. TIRANT LO BLANCH.

Barnes, C. (2010). Discapacidad, política y pobreza en el contexto del mundo mayoritario. Política y Sociedad, 47(1),11-25.

Bernabeu, E. (2012). The social model analysis of disability and the majority world. Revista Sociológica de Pensamiento Crítico, 6(2), 279-288.
Brogna, P. (2012). La condición del adulto con discapacidad intelectual. Posición social y simbólica del otro. (tesis doctoral). Universidad Nacional Autónoma de México, México.

Camacho, C. \& Varela, G. (2011). Inclusión educativa virtual de estudiantes con discapacidad motora. Apertura, (11), 44-57, Recuperado https://www.redalyc.org/articulo.oa?id=688/68826916005

CEPAL \& CAF (2020). Las oportunidades de la digitalización en América Latina frente al Covid-19. Naciones Unidas. Recuperado de: https://www.cepal.org/es/publicaciones/45360oportunidades-la-digitalizacion-america-latina-frente-al-covid19

CEPAL. (2014). Informe regional sobre la medición de la discapacidad. Una mirada a los procedimientos de medición de la discapacidad en América latina y el Caribe. Comisión Económica para América Latina y el Caribe (CEPAL). Recuperado de: https://repositorio.cepal.org/handle/11362/36906

Comisión Económica para América Latina y el Caribe (2018). La Agenda 2030 y los Objetivos de Desarrollo Sostenible: una oportunidad para América Latina y el Caribe. Naciones Unidas. Recuperado de: http://www.cepal.org

Comisión Económica para América Latina y el Caribe (2020). La pandemia del COVID-19 profundiza la crisis de los cuidados en América Latina y el Caribe.Informe especial COVID-19. Naciones Unidas. Recuperado de: http://www.cepal.org

Comisión Económica para América Latina y el Caribe (CEPAL) (2018). Agenda 2030 y los Objetivos de Desarrollo Sostenible Una oportunidad para América Latina y el Caribe. Naciones Unidas. Recuperado de: http://www.cepal.org

Díaz, E. (2018). La investigación social en la esfera de la discapacidad: realidad y tendencias. Revista Española de Discapacidad, 6 (II), 191-203.

Ferrante, C. \& Ferreira, M. (2010). El habitus de la discapacidad: la experiencia corporal de la dominación en un contexto económico periférico. Política y Sociedad, 47(1), 85-104

Flórez, L., Ramírez, C. \& Ramírez, S. (2016). Las TIC como herramientas de inclusión social.3C TIC, 5(1),55-67. Recuperado de: $\quad$ https://www.3ciencias.com/wpcontent/uploads/2016/03/LAS-TIC-COMO-HERRAMIENTASDE-INCLUSI\%C3\%93N-SOCIAL.pdf

García, I.C. \& Tamayo, J. (2018). Las políticas educativas inclusivas en Cuba. Revista Científico-Metodológica, Edición especial, 1-9.

Gómez, J.C., López, D. \& Velásquez, C.M. (2006). La naturaleza de la comunicación: un aporte a su discusión conceptual. Palabra clave, 9(1), 143-167.

González, R. (2010). Política social y organizaciones en torno a la discapacidad. Editoriales Once Ríos.

Habermas, J. (2012). La inclusión del otro. Estudios de teoría política. Paidós.

Huenchuan, S. (2020). El derecho a la vida y la salud de las personas mayores el marco de la pandemia por COVID-19. Comisión Económica para la América Latina y el Caribe. 
Personas con Discapacidad y Aprendizaje Virtual: Retos para las TIC en Tiempos de Covid-19.

Jiménez, A. \& Huete, A. (2010). Estadísticas y otros registros sobre discapacidad en España. Política y Sociedad, 47(1), 165-173

Lancheros, D., Carillo, A., \& Lara, J. (2011). Modelos de adaptación en ambientes virtuales de aprendizaje para personas con discapacidad. Revista Avances en Sistemas e Informática, 8(2), $17-30$

Mogollón, I., Medina, C., \& Correa, K. (2017). Desarrollo de experiencias de aprendizaje virtual accesible. Atención a las necesidades de personas con discapacidad visual. EDUTEC. Revista Electrónica de Tecnología Educativa. (62). dx.doi.org/10.21556/edutec.2017.62.1023

Organización de Naciones Unidas. (2011). Disability and the Millennium Development Goals, A Review of the MDG Process and Strategies for Inclusion of Disability Issues in Millennium Development Goal Efforts. Recuperado de: http://www.un.org/disabilities/documents/review_of_disability_ and_the_mdgs.pdf

Organización Mundial de la Salud (2011). Informe mundial de la discapacidad. Recuperado de: http://cdrwww.who.int/entity/disabilities/world_report/2011/su mmary_es.pdf.

Palacios, A. \& Barrifi, F. (2007). La discapacidad como una cuestión de derechos humanos. Una aproximación a la Convención Internacional sobre los derechos de las personas con discapacidad. Ediciones Cinca.

Palacios, A. \& Romañach, J. (2006). El modelo de la diversidad. La Bioética y los Derechos Humanos como herramientas para alcanzarla plena dignidad en la diversidad funcional. Ediciones Diversitas- AIES.

Pérez, L. (2010). Discapacidad, Derecho y Políticas de Inclusión. Ediciones Cinca.

Pérez, M. y Chhabra, G. (2019). Modelos teóricos de discapacidad: un seguimiento del desarrollo histórico del concepto de discapacidad en las últimas cinco décadas. Revista Española de Discapacidad, 7 (I), 7-27.

Renzaglia, A. et al. (2003). Promoting a lifetime of inclusion. Focus on Autism and Other Developmental Disabilities, 18 (3),140-149.

Samaniego, P. (2006). Aproximación a la realidad de las personas con discapacidad en Latinoamérica. Comité Español de Representantes de Personas con Discapacidad (CERMI).

Silvana, P. \& Andrea, M. (2016). Hacia las tecnologías para la inclusión social en contextos educativos regionales: análisis del caso ECCA. Revista Electrónica Actualidades Investigativas en Educación, 16(2), 1-26. http://dx.doi.org/10.15517/aie.v16i2.23564

Subirats, J., Alfama, E., \& Obradors, A. (2009). Ciudadanía e inclusión social frente a las inseguridades contemporáneas. La significación del empleo. Instituto de Gobierno y Políticas Públicas.

Venturiello, M. (2017). Políticas sociales en discapacidad: una aproximación desde las acciones del Estado en Argentina. Revista Española de Discapacidad, 5 (2), 149-169. 